$$
\text { CONF-9607101--1 }
$$

\title{
WHEEL ROLLING CONSTRAINTS AND SLIP IN MOBILE ROBOTS*
}

\author{
Shashank Shekhar \\ Robotics and Process Systems Division \\ Oak Ridge National Laboratory \\ P.O. Box 2008 \\ Oak Ridge, TN 37381-6305
}

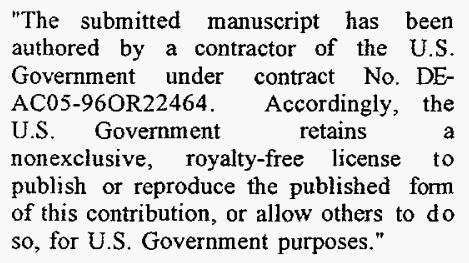

Submitted to: Second International Workshop on Algorithmic Foundation of Robotics, LAAS-CNRS, Toulouse, France, July 3-5,1996

\footnotetext{
*This research was supported in part by the U.S. Air Force Material Command (AFMC) San Antonio Air Logistics Center, Robotics and Automation Center of Excellence (SA/ALC-RACE) under Interagency Agreement 2146-H055-Al with the U.S. Department of Energy, under Interagency Agreement 2072-E123-A1 with the U.S. Department of Energy, under contract number DE-AC05-960R22464 with Lockheed Martin Energy Research Corp., and in part by an appointment to the Oak Ridge National Laboratory Postdoctoral Research Associates Program administered jointly by the Oak Ridge National Laboratory and the Oak Ridge Institute of Science and Education.
} 


\section{DISCLAIMER}

Portions of this document may be illegible in electronic image products. Images are produced from the best available original document. 


\title{
Wheel Rolling Constraints and Slip in Mobile Robots
}

\author{
Shashank Shekhar \\ Robotics and Process Systems \\ Oak Ridge National Laboratory \\ Oak Ridge, TN 37831-6305, USA \\ ShekharS@ornl.gov
}

February 9, 1996

\begin{abstract}
It is widely accepted that dead-reckoning based on the rolling with no-slip condition on the wheels is not a reliable method to ascertain the position and orientation of a mobile robot for any reasonable distance. We establish that wheel slip is inevitable under the dynamic model of motion using classical results on the accessibility and controllability in nonlinear control theory and an analytical model of rolling of two linearly elastic bodies.
\end{abstract}

\section{Introduction}

The mobility literature of wheeled mobile robots with fixed, centered, off-centered and omni-directional wheels is traditionally founded in the equations of motion derived from the rolling with no-slip constraint on the wheels. Together with the wheel orientation encoders that are used to infer the configuration (end-point) of the mobile robot, these constraints are convenient in reducing the order of the state-space description of the mobile robot. However, dead-reckoning error is substantial for large distances. It renders the reduced state-space model and the corresponding dead-reckoning method of inferring the configuration of the mobile robot, at best, questionable.

Our objective in this paper is to explore the implications of imposing the rolling with no-slip condition using classical results on the accessibility and controllability in nonlinear control theory [NV 90]. When the rolling constraints are imposed, they allow arbitrarily large forces to be transmitted with perfect rolling contact. The analytical theory of two bodies in rolling contact, however, establishes a definite slip associated with the traction forces at the wheel-ground interface. We consider that the traction forces at the wheel-ground interface is determined under the following conditions:

\section{Hypothesis 1 .}

a. The rolling bodies are linearly elastic,

b. Quasi-identity relation on the elastic properties of the two bodies in contact holds (this includes the case when the two bodies are elastically similar and approximates the situation when one body, say a rubber wheel, is incompressible, and the other body, say the concrete ground, is relatively rigid.)

c. The area of contact between the two bodies is symmetric about the direction of the rolling of the wheels.

The conditions we identify are roughly the following: 
1. If the constraints in the lateral (sideways) and longitudinal (rolling) directions of a wheel transmit traction forces determined under the conditions of the Hypothesis 1 of rolling contact, then only mobile robots with off-centered wheels can, in general, preserve the kinematic constraints imposed by the wheels. The state of rest is, however, an equilibrium point of the dynamic system,

2. If the lateral (sideways) direction of the wheels transmit arbitrarily large forces, then preserving the longitudinal direction constraint of rolling with no-slip with wheel-gound traction determined under the conditions of the Hypothesis 1 implies that the base of those mobile robots with fixed, centered, and omni-directional wheels can not change its state from the state of rest - a case of zero accessibility (and controllability) for the base of the mobile robot.

In effect, we identify conditions for which wheel-slip is inevitable. A key aspect of our study is a review of some results in the analytical theory of rolling of two linearly elastic bodies in contact. The origin of such studies is founded in the law of friction of Coulomb-Amontons and the analytic models of deformation of a three-dimensional half-space elastic body due to a concentrated load of Boussinesq (1885), Cerruti (1882) and Hertz's Theory (1882) of two elastic surfaces with curvature in contact. Application of these theories to the study of rolling contact between two bodies was initiated by Carter (1926) who gave solutions of a two-dimensional problem, i.e. when the extents of the rolling objects lie in a plane. Subsequently, Fromm (1927), Johnson (1958), de Pater (1956), Kalker (1957), Haines and Ollerton (1964), and Heinrich and Desoyer (1967) have extended the solutions under various other assumptions; see a review article by Kalker [Kalker 79].

Previously, Alexander and Maddocks [AM 89] considered wheel scrubbing. It arises from inconsistent positioning and orientation of the wheels with respect to kinematic mobility. They also offer an analytical justification of the phenomena of sideways lurching with uneven rolling friction conditions on the wheels using a minimum work principle on their quasi-static model of motion. The wheel slip we consider here and the implication on its existence subsumes kinematic consistency. It is, therefore, different from their wheel scrubbing phenomena. A recent paper of Balakrishna and Ghosal [BG 95] considered a model of the traction forces arising from a rolling tire. Their model of traction force and wheel slip arises from an empirical model of the tire mechanics analysis. An analytical model of a rolling tire, in the sense we present here for two linearly elastic rolling body, is a difficult problem [Kalker 90]. However, since their empirical model incorporates essential aspects of the analytical theory of rolling under Hypothesis 1 that we consider, their numerical simulation results exhibit the presence of wheel slip - a conclusion we prove based entirely on an analytical theory. Our primary results are Theorems 6 and 8 [Sh 96].

\section{Kinematics}

This section introduces the kinematic constraints imposed by the nature and configuration of various types of wheels of the mobile robot. First, we consider the transformation of kinematic quantities in a general framework of the choice of co-ordinate systems. The sub-sections following the first consider the form of the specific instances of the kinematic constraints of a wheeled mobile robot.

\subsection{Kinematic Transformation}

The set of rigid body motion in a space $\mathcal{S}$ is called Special Orthogonal Euclidean Group denoted $S E(n)$. An element $\mathbf{q} \in S E(n)$ is a map $\mathbf{q}: \mathcal{S} \rightarrow \mathcal{S}: \mathbf{p} \mapsto \mathbf{q}(\mathbf{p})$ that preserves the norm. Consider also a choice of co-ordinate system on the space $\mathcal{S}$ as an assignment of $n$ real-valued norm-preserving orthogonal map $\mathbf{x}: \mathcal{S} \rightarrow \mathcal{R}^{n}: \mathbf{p} \stackrel{\mathbf{x}}{\mapsto}\left(x^{1}(\mathbf{p}), \ldots, x^{n}(\mathbf{p})\right)$. Given a choice of co-ordinate system on a space $\mathcal{S}$, an element of $S E(n)$ has several prevalent representation including homogeneous matrix transformation and Plüker Screw Coordinates. Consider an element $\mathbf{q} \in S E(n)$ and its representation $\mathbf{q}^{\mathbf{x}}$ in the choice of a coordinate system $\mathbf{x}$ such that $\mathbf{q}^{\mathbf{x}}(\mathbf{p})=\mathbf{x} \circ \mathbf{q} \circ \mathbf{x}^{-1}(\mathbf{p})$ where $\circ$ represents map composition operator. A 
representation of an element $\mathbf{q}^{\mathbf{X}}$ in the homogeneous matrix transformation is a linear transformation

$$
\mathbf{q}^{\mathbf{x}}=\left[\begin{array}{cc}
\mathbf{R}^{\mathbf{x}} & \mathbf{t}^{\mathbf{x}} \\
0 & 1
\end{array}\right]
$$

where $\mathbf{R}^{\mathbf{X}}$ is a $n \times n$ matrix representing rotation and $\mathbf{t}^{\mathbf{X}}$ is a $n$-dimensional translational vector both expressed in the choice of co-ordinate system $\mathbf{x}$ so that $\mathbf{q}(\mathbf{p})=\mathbf{x}^{-1}\left(\mathbf{R}^{\mathbf{x}} \mathbf{x}(\mathbf{p})+\mathbf{t}^{\mathbf{x}}\right)$ for all points $\mathbf{p} \in \mathcal{S}$. The elements $\mathbf{R}^{\mathbf{X}}$ and $\mathbf{t}^{\mathbf{x}}$, called rotations and translations respectively, are representations of $\mathbf{R}$ and $\mathbf{t}$, members of the space $S E(n)$, in the choice of co-ordinate system $\mathbf{x}$. In general, an arbitrary element $\mathbf{q} \in S E(n)$ is composed of a rotation and translation, i.e. $\mathbf{q}=\mathbf{t} \mathbf{q} \circ \mathbf{R} \mathbf{q}$.

Another choice of a co-ordinate system on the space $\mathcal{S}$, say $\mathbf{y}$, induces different representation of an element $\mathbf{q} \in S E(n)$. Let the two choices of co-ordinate systems $\mathbf{x}$ and $\mathbf{y}$ be related by a map $\mathbf{h} \in S E(n)$, such that $\mathbf{y}=\mathbf{x} \circ \mathbf{h}^{-1}$, then two representaions of an element $\mathbf{q} \in S E(n)$ are related by

$$
\mathbf{q}^{\mathbf{y}}=\mathbf{h}^{-1} \mathbf{x} \circ \mathbf{q}^{\mathbf{x}} \circ \mathbf{h}^{\mathbf{x}}
$$

Consider the space of infinitesimal rigid body motions at the identity $e \in S E(n)$, the initial configuration. The space denoted $s e(n)$ is the tangent space $T_{e} S E(n)$ of the manifold $S E(n)$ at the identity. Any choice of co-ordinate system $\mathbf{x}$ on the space also induces a representation of an element $\mathbf{v}_{e}$ in $s e(n)$ denoted $\mathbf{v}_{e}^{\mathbf{X}}$. For example, $\mathbf{v}_{e}^{\mathbf{x}} \in \operatorname{se}(3), \mathbf{v}_{e}^{\mathbf{X}}=\left[\begin{array}{llllll}v_{e_{1}}^{\mathbf{X}} & v_{e_{2}}^{\mathbf{X}} & v_{e_{3}}^{\mathbf{X}} & \omega_{e_{1}}^{\mathbf{X}} & \omega_{e_{2}}^{\mathbf{X}} & \omega_{e_{3}}^{\mathbf{X}}\end{array}\right]^{T}$, where $\left[\begin{array}{lll}v_{e_{1}}^{\mathbf{X}} & v_{e_{2}}^{\mathbf{X}} & v_{e_{3}}^{\mathbf{X}}\end{array}\right]^{T}$ is the translational velocity and $\left[\begin{array}{lll}\omega_{e_{1}}^{\mathbf{X}} & \omega_{e_{2}}^{\mathbf{X}} & \omega_{e_{3}}^{\mathbf{X}}\end{array}\right]^{T^{T}}$ is the angular velocity both expressed in the choice of co-ordinate system $\mathbf{x}$.

Another choice of coordinate system, say $\mathbf{y}$, on the space $\mathcal{S}$ induces a map of an element $\mathbf{v}_{e} \in \operatorname{se}(n)$ from one represenation to the other. For a given element $\mathbf{h} \in S E(n)$, define the adjoint as a map $A d_{\mathbf{h}}: s e(n) \rightarrow s e(n)$. For example, if $\mathbf{h}$ is represented as a homogeneous transformation matrix in the coordinate system $\mathbf{x}$ with $\mathbf{R}^{\mathbf{h}^{\mathbf{X}}}$ as the rotation matrix and $\mathbf{t}^{\mathbf{h}}$ as the translation vector, then the adjoint $A d_{\mathbf{h}}$ is represented as

$$
A d_{\mathbf{h}}^{\mathbf{x}}=\left[\begin{array}{cc}
\mathbf{R}^{\mathbf{h}^{\mathbf{x}}} & {\left[\mathbf{t}^{\mathbf{h}^{\mathbf{x}}}\right] \mathbf{R}^{\mathbf{h}^{\mathbf{x}}}} \\
0 & \mathbf{R}^{\mathbf{h}^{\mathbf{x}}}
\end{array}\right]
$$

where $[\mathbf{t}]$ represents the cross-product operator of the vector $\mathbf{t}=\left(t_{1}, t_{2}, t_{3}\right)$ defined for $n=3$ as

$$
[\mathbf{t}]=\left[\begin{array}{ccc}
0 & -t_{3} & t_{2} \\
t_{3} & 0 & -t_{1} \\
-t_{2} & t_{1} & 0
\end{array}\right]
$$

The representations of $\mathbf{v}_{e}$ in coordinate system $\mathbf{x}$ and $\mathbf{y}$ are related as

$$
\mathbf{v}_{e}^{\mathbf{y}}=A d_{\mathbf{h}-1}^{\mathbf{x}}\left(\mathbf{v}_{e}^{\mathbf{x}}\right) \text {. }
$$

\subsection{Kinematic Model of Motion of the Base of Wheeled Mobile Robot}

First consider the configuration of the base of the wheeled mobile robot in the plane of motion. Let $\mathcal{F}$ be a choice of a co-ordinate system in the plane of motion denoted $\mathcal{S}$ so that $\mathcal{F}: \mathcal{S} \rightarrow \mathcal{R}^{2}: \mathrm{p} \stackrel{\mathcal{F}}{\mapsto}$ $(x(\mathbf{p}), y(\mathbf{p}))$. Let the configuration of the base of the mobile robot, an element of $S E(2)$, be denoted $\mathbf{x}_{1}=(x, y, \theta)$ in the choice of co-ordinate system $\mathcal{F}$. The rigid body configuration $\mathbf{x}_{1}$ is also equivalent to $\mathbf{t}(x, y) \circ \mathbf{R}(\theta)=\mathbf{t}\left(\mathbf{x}_{1}\right) \circ \mathbf{R}\left(\mathbf{x}_{1}\right)^{1}$. It is easy to verify that a choice of co-ordinate $\mathcal{M}=\mathbf{x}_{1}^{-1} \circ \mathcal{F}$ is fixed to the base of the mobile robot. Lets call $\mathcal{M}$ the moving reference frame.

Let the velocity of the base of the mobile robot in the plane of motion at the configuration $\mathbf{x}_{1}$ be denoted $\dot{\mathbf{x}}_{1}=(\dot{x}, \dot{y}, \dot{\theta})$. It is easy to verify that the velocity of the base of the mobile robot $\dot{\mathbf{x}}_{1}^{\mathcal{M}}$ in

\footnotetext{
${ }^{1}$ Items in () denote, depending on the context, elements of a vector or functional dependency of a map on variables. If $f: \mathcal{R}^{n} \rightarrow \mathcal{R}^{m}$ is smooth map such that $f=\left(f^{1}, f^{2}, \ldots, f^{m}\right)$, then $\frac{\partial f^{i}\left(x_{1}, x_{2}, \ldots, x_{n}\right)}{\partial x_{j}} \equiv D_{j} f^{i} \equiv D f[i][j]$, and $D f \equiv \frac{\partial f}{\partial \mathbf{X}}$ is the Jacobian of the function $f$.
} 


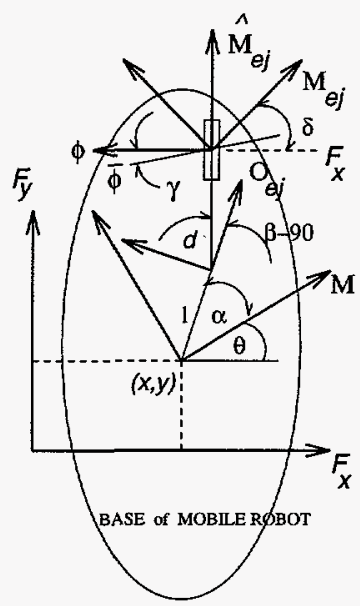

Figure 1: Choice co-ordinate systems for kinematic constraints.

the moving reference frame is related to $\dot{x}_{1}$ by $\dot{\mathbf{x}}_{1}^{\mathcal{M}}=\operatorname{Ad}_{\left(\mathbf{R}^{\left.\mathbf{x}_{1}\right)^{-1}}\right.}^{\mathcal{I}}\left(\dot{\mathbf{x}}_{1}\right)$. More specifically, this relation reduces to

$$
\dot{\mathbf{x}}_{1}^{\mathcal{M}}=\hat{\mathbf{R}} \dot{\mathbf{x}}_{1}
$$

where $\hat{\mathbf{R}}=\hat{\mathbf{R}}\left(\mathbf{x}_{1}\right)$ is an orthogonal tranformation matrix of three-by-three of the form

$$
\hat{\mathbf{R}}(\theta)=\left[\begin{array}{cc}
\mathbf{R}^{\mathbf{x}_{1}} & 0 \\
0 & 1
\end{array}\right]=\left[\begin{array}{ccc}
\operatorname{Cos}(\theta) & \operatorname{Sin}(\theta) & 0 \\
-\operatorname{Sin}(\theta) & \operatorname{Cos}(\theta) & 0 \\
0 & 0 & 1
\end{array}\right] .
$$

\subsection{Kinematic Constraints Imposed by Wheels}

Our model of a wheeled mobile robot is a generalized model of such robots considered by Campion et. al. [CBD 93]. A wheeled mobile robot has either a conventional type of wheels or an omnidirectional type wheel. A conventional type wheel has a given axis about which the wheel can rotate and is driven. It is of the following three categories: (i) fixed, (ii) centered orientable, and (iii) off-centered orientable. An omni-directional wheel can rotate about an arbitrary axis of rotation in the plane of motion of the base of the mobile robot and is (usually) driven about one given axis in that plane. The configuration of a mobile robot with an arbitrary combination of wheels is described by the following configuration elements in addition to the three co-ordinates of the base $\mathbf{x}_{1}: \mathbf{x}_{2}$, the vector of angular orientations of the plane containing the off-centered wheel, $\mathbf{x}_{3}=\left(\phi_{f}, \phi_{c}, \phi_{o c}, \phi_{o d}\right)$, the angular orientations of the fixed, centered, off-centered, and omni-directional wheels respectively about their driven directions, $\mathbf{x}_{4}=\left(\bar{\phi}_{\text {od }}\right)$, an appropriate choice of angular velocities of the omni-directional wheels about directions complimentary to the directions of their drive, $\mathbf{x}_{5}$, the orientation of the plane containing centered wheels. If the number of fixed, centered, off-centered, and omni-directional wheels are $N_{f}, N_{c}, N_{o c}$, and $N_{\text {od }}$ respectively, then the dimension of an element $\mathbf{x}_{c}=\left(\mathbf{x}_{1}, \mathbf{x}_{2}, \mathbf{x}_{3}, \mathbf{x}_{4}, \mathbf{x}_{5}\right)$ describing the configuration of the mobile robot is $3+N_{o c}+\left(N_{f}+N_{c}+N_{o c}+N_{o d}\right)+N_{o d}+N_{c}=3+N_{f}+2\left(N_{c}+N_{o c}+N_{o d}\right)$. The kinematic model of a wheeled mobile robot is derived using an idealized wheel model - a zero width non-deformable planar circle rolling with no-slip on the ground. Let $\mathcal{O}_{e j}, e \in\{f, c, o c, o d\}$, $j \in\left\{1, \ldots, N_{e}\right\}$ be the choice of co-ordinate frame of the $j^{t h}$ wheel of type $e$ with the origin on the wheel axle above the point of wheel-ground contact for fixed, centered, and omni-directional wheels and at the pivot of the arm of off-centered wheels. Let $\mathbf{a}_{e j}$ be the representation in the choice of co-ordinate frame $\mathcal{M}$ such that $\mathcal{O}_{e j}=\mathbf{a}_{e j}^{-1} \circ \mathcal{M}$. Let $\mathcal{M}_{e j}, e \in\{f, c, o c, o d\}, j \in\left\{1, \ldots, N_{e}\right\}$ be the slip coordinate frames with the origin on the wheel axle above the point of wheel-ground contact for all wheels so that $\mathcal{M}_{e j}=\mathbf{h}_{e j}^{-1} \circ \mathcal{O}_{e j}$, where $\mathbf{h}_{e j}$ is the representation in the choice of co-ordinate frame $\mathcal{O}_{e j}$ relating the frame $\mathcal{O}_{e j}$ and the slip co-ordinate frame $\mathcal{M}_{e j}$. The maps $\mathbf{h}_{e j}$ may be identity for fixed, centered, and 
omni-directional wheels but relates the point on the wheel axle to the pivot of the off-centered arms on the base of the mobile robot. The velocity of a point on the axle of the wheels above the point of wheel-ground contact in their slip co-ordinate frames $\mathcal{M}_{e j}$ is $\dot{\mathbf{x}}_{1}^{\mathcal{M}_{e j}}=\operatorname{Ad}_{\mathbf{h}_{e j}} \operatorname{Ad}_{\mathbf{a}_{e j}} \hat{\mathbf{R}} \dot{\mathbf{x}}_{1}+\operatorname{Ad}_{\mathbf{h}_{e j}}(0,0, \dot{\beta})$ where $\dot{\beta}=\left(\dot{\mathbf{x}}_{2}\right)_{j}$ for the $j^{\text {th }}$ off-centered wheel and zero otherwise.

Let $\hat{\mathcal{M}}_{e j}, e \in\{f, c, o c, o d\}, j \in\left\{1, \ldots, N_{e}\right\}$ be the wheel coordinate frames with origin at the wheelground contact so chosen that $\mathbf{v}_{w}$, the velocity of the mobile robot due to the wheel's rolling with no-slip condition and no relative angular motion at the wheel-ground contact are expressed conveniently. Let $\mathcal{M}_{e j}=\mathrm{g}_{e j}^{-1} \circ \hat{\mathcal{M}}_{e j}$ relate the slip co-ordinate frame and the wheel co-ordinate frame where $\mathrm{g}_{e j}$ is expressed in the $\hat{\mathcal{M}}_{e j}$ choice of co-ordinate frame.

The transformation $\mathbf{h}_{e j}$ 's are chosen such that the $y$-component of the velocity $\dot{\mathbf{x}}_{1}^{\mathcal{M}_{e j}}$ is zero. In other words, the slip co-ordinate frames are chosen so their $x$-axis is aligned with the direction of the velocity of the axle of the wheel at the point of wheel-ground contact. In addition to the parameters that determine the wheel-ground contact point in relation to the moving frame of reference $\mathcal{M}$, this choice makes the transformations $\mathbf{h}_{e j}$ 's dependent on $\dot{\mathbf{x}}_{1}$, and depending on the type of the wheel, also on $\dot{\mathbf{x}}_{2}$. When $\dot{\mathbf{x}}_{1}^{\mathcal{M}_{e j}}$ is zero, this choice is arbitrary. The zero of the function

$$
\operatorname{Ad}_{\mathbf{h}_{e j}} \operatorname{Ad}_{\mathbf{a}_{e j}} \hat{\mathbf{R}} \dot{\mathbf{x}}_{1}+\operatorname{Ad}_{\mathbf{h}_{e j}}(0,0, \dot{\beta})-\operatorname{Ad}_{\mathbf{g}_{e j}^{-1}}\left(\mathbf{v}_{w}\right)
$$

defines the kinematic constraints on the motion of the base of the mobile robot due to the $j^{\text {th }}$ wheel of the type $e \in\{f, c, o c, o d\}$ with wheels rolling with no-slip condition. These constraints incorporate motion due to the swing of the arm of the off-centered wheels and no relative angular motion of the wheels with respect to the ground at the point of wheel-ground contact (see Appendix for a specific instantiation of these constraints).

The three scalar constraints in equation (4) for each wheel restrict the motion of the base of the mobile robot at the point of wheel-ground contact in the $x, y$, and $\theta$ directions of the slip co-ordinate system $\mathcal{M}_{e j}$. For convenience, the slip co-ordinate frame $x$-direction will also be called the longitudinal direction, the $y$-direction as the lateral direction, and the $\theta$-direction as the rotational direction. In this terminology, the scalar kinematic constraints for each wheel are also called longitudinal, lateral and rotational constraints due to the $e j^{\text {th }}$-wheel. The constraints in the longitudinal direction impose rolling with no-slip condition on the wheels of the mobile robot. The constraints in the lateral and rotational directions impose no-lateral and no-rotational slips respectively. There are $\left(N_{f}+N_{c}+N_{o c}+N_{o d}\right)$ constraints of the longitudinal, lateral, and rotational type. Let the longitudinal, lateral, and rotational constraints for all the wheels be collected in the form $\mathbf{J}_{x} \dot{\mathbf{x}}_{\mathfrak{c}}, \mathbf{J}_{y} \dot{\mathbf{x}}_{\mathfrak{c}}$, and $\mathbf{J}_{\theta} \dot{\mathbf{x}}_{c}$ respectively, where

$$
\begin{aligned}
& \mathbf{J}_{x}=\left[\begin{array}{cc}
\mathbf{J}_{x 1 f}\left(\mathbf{x}_{1}, \dot{\mathbf{x}}_{1}\right) & 0 \\
\mathbf{J}_{x 1 c}\left(\mathbf{x}_{1}, \mathbf{x}_{5}, \dot{\mathbf{x}}_{1}\right) & 0 \\
\mathbf{J}_{x 1 o c}\left(\mathbf{x}_{1}, \mathbf{x}_{2}, \dot{\mathbf{x}}_{1}, \dot{\mathbf{x}}_{2}\right) & \mathbf{J}_{x 2 o c}\left(\mathbf{x}_{1}, \mathbf{x}_{2}, \dot{\mathbf{x}}_{1}, \dot{\mathbf{x}}_{2}\right) \\
\mathbf{J}_{x 1 \text { od }}\left(\mathbf{x}_{1}, \dot{\mathbf{x}}_{1}\right) & 0
\end{array}\right.
\end{aligned}
$$

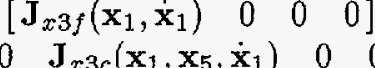

$$
\begin{aligned}
& {\left[\begin{array}{llll}
0 & 0 & \mathbf{J}_{x 3 o c}\left(\mathbf{x}_{1}, \mathbf{x}_{2}, \dot{\mathbf{x}}_{1}, \dot{\mathbf{x}}_{2}\right) & 0
\end{array}\right]} \\
& {\left[\begin{array}{llll}
0 & 0 & 0 & \mathbf{J}_{x 3 o d}\left(\mathbf{x}_{1}, \dot{\mathbf{x}}_{1}\right)
\end{array}\right]} \\
& \mathbf{J}_{y}=\left[\begin{array}{cc}
\mathbf{J}_{y 1 f}\left(\mathbf{x}_{1}, \dot{\mathbf{x}}_{1}\right) & 0 \\
\mathbf{J}_{y 1 c}\left(\mathbf{x}_{1}, \mathbf{x}_{5}, \dot{\mathbf{x}}_{1}\right) & 0 \\
\mathbf{J}_{y 1 o c}\left(\mathbf{x}_{1}, \mathbf{x}_{2}, \dot{\mathbf{x}}_{1}, \dot{\mathbf{x}}_{2}\right) & \mathbf{J}_{y 2 o c}\left(\mathbf{x}_{1}, \mathbf{x}_{2}, \dot{\mathbf{x}}_{1}, \dot{\mathbf{x}}_{2}\right) \\
\mathbf{J}_{y 1 a d}\left(\mathbf{x}_{1}, \dot{\mathbf{x}}_{1}\right) & 0
\end{array}\right. \\
& {\left[\mathbf{J}_{y 3 f}\left(\mathbf{x}_{1}, \dot{\mathbf{x}}_{1}\right) \quad 0 \quad 0 \quad 0\right]}
\end{aligned}
$$

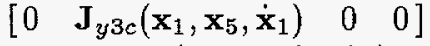

$$
\begin{aligned}
& {\left[\begin{array}{llll}
0 & 0 & \mathbf{J}_{y 3 a c}\left(\mathbf{x}_{1}, \mathbf{x}_{2}, \dot{\mathbf{x}}_{1}, \dot{\mathbf{x}}_{2}\right) & 0
\end{array}\right]} \\
& {\left[\begin{array}{llll}
0 & 0 & 0 & \mathbf{J}_{y 3 \circ d}\left(\mathbf{x}_{1}, \dot{\mathbf{x}}_{1}\right)
\end{array}\right]}
\end{aligned}
$$

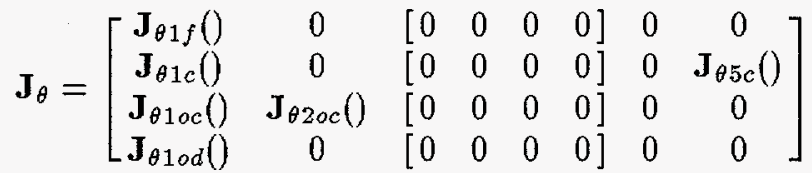

$$
\begin{aligned}
& \mathbf{J}_{x 4 \circ d}\left(\mathbf{x}_{1}, \dot{\mathbf{x}}_{1}\right)
\end{aligned}
$$

and constraints in the fourth column due to the angular velocity of the wheels are further expanded into four sub-components corresponding to the fixed, centered, off-centered, and omni-directional wheels. The functional dependency of each of the terms in the Jacobians $\mathbf{J}_{x}, \mathbf{J}_{y}$, and $\mathbf{J}_{\theta}$ as indicated above are based on certain idealization of the wheels. 


\section{Rolling Contact of Two Elastic Bodies}

This section reviews existing results in Contact Mechanics on two elastic bodies rolling in contact with each other. We show certain symmetry in the analytical creep-force relation and review other symmetries given by Kalker [Kalker 67] with the elastic quasi-identity assumption of the Hypothesis 1. These relations allow us to infer the traction force at zero slip velocity.

\subsection{Creep-Force Relation Problem Definition}

Consider a linearly elastic circular body, denoted $e j$, rolling on a planar linearly elastic material. Let $\dot{\mathbf{x}}_{1}^{\mathcal{M}_{e j}}$, the velocity of the center of the wheel axle be in the $x$-direction of a co-ordinate system $\mathcal{M}_{e j}$ chosen in the plane of motion of the wheel with the origin at the point of wheel-ground contact - the slip co-ordinate frames defined in Section 2.3. Let $\mathbf{J}_{\{x, y, \theta\} e j}$ refer to the $j^{\text {th }}$ row in the $e^{\text {th }}$ type row block of the Jacobians $\mathbf{J}_{x}, \mathbf{J}_{y}$, or $\mathbf{J}_{\theta}$ defined in equations (5), (6), and (7) respectively. The terms $\mathbf{J}_{\{x, y, \theta\} e j} \dot{\mathbf{x}}_{c}$ represent the rigid slip of the wheel at the wheel ground interface in the $x, y$, and $\theta$ directions respectively of the slip co-ordinate frames. Define $\nu_{x e j}$, the longitudinal creepage, $\nu_{y e j}$, the lateral creepage, and $\nu_{\theta e j}$, the spin for the wheels as

$$
\nu_{\{x, y, \theta\} e j}=\frac{\mathbf{J}_{\{x, y, \theta\} e j} \dot{\mathbf{x}}_{c}}{V_{e j}}
$$

where $V_{e j}=\left|\dot{x}_{1\{x, y\}}^{\mathcal{M}_{e j}}\right|$ is the magnitude of the $x$ and $y$, the translational, velocities of the point on the axle of the wheel. The creepage and spin are ratios of the rigid wheel slip to the magnitude of the translational velocity of the axle of a wheel.

Let the area of contact of the wheel with the ground be denoted $C_{e j}$ described in the respective slip co-ordinate frames. The material in the two bodies in the area of contact deform elastically due to the friction induced tangential traction and the vertical load induced compression. Let the slip co-ordinate frames be the choice of the co-ordinate system to describe the contact area $C_{e j}$. Let us add $z$-axis to the slip frames so that the $z$-positive direction points into the material of the wheel. In this description, the two bodies are approximated as half-spaces with the material on $z \geq 0$ and $z \leq 0$ of the slip coordinate frames. The elastic deformation on these half-spaces due to concentrated normal load in the $z$-direction and tangential load along the $x$-axis and the $y$-axis have been given by Boussinesq (1885) and Cerruti (1882) [Love 44]. Let the elastic strain denoted $\mathbf{u}_{e j}\left(x, y, \nu_{x e j}, \nu_{y e j}, \nu_{\theta e j}\right)=\left(u_{x e j}, u_{y e j}, u_{z e j}\right)$ be the difference in the elastic strains of the rolling wheel and the ground expressed in the slip coordinate frame. Let $\left(X_{e j}, Y_{e j}, Z_{e j}\right)\left(x, y, \nu_{x e j}, \nu_{y e j}, \nu_{\theta e j}\right)$ be the $x, y$ and $z$ components of the traction and the vertical load at a point $(x, y) \in C_{e j}$. The relation between the material strain function $\mathbf{u}$ and the traction $(X, Y, Z)$ is

$$
\left[\begin{array}{l}
u_{x e j} \\
u_{y e j} \\
u_{z e j}
\end{array}\right]=\frac{1}{\pi G} \iint_{C_{e j}}\left[\begin{array}{ccc}
\frac{1-\sigma}{R}+\frac{\sigma\left(x-x^{*}\right)^{2}}{R^{3}} & \frac{\sigma\left(x-x^{*}\right)\left(y-y^{*}\right)}{R^{3}} & -\kappa \frac{\left(x-x^{*}\right)}{R^{2}} \\
\frac{\sigma\left(x-x^{*}\right)\left(y-y^{*}\right)}{R^{3}} & \frac{1-\sigma}{R}+\frac{\sigma\left(y-y^{*}\right)^{2}}{R^{*}} & -\kappa \frac{\left(y-y^{*}\right)}{R^{2}} \\
\kappa \frac{\left(x-x^{*}\right)}{R^{2}} & \kappa \frac{\left(y-y^{*}\right)}{R^{2}} & \frac{1-\sigma}{R}
\end{array}\right]\left[\begin{array}{c}
X_{e j}\left(x^{*}, y^{*}\right) \\
Y_{e j}\left(x^{*}, y^{*}\right) \\
Z_{e j}\left(x^{*}, y^{*}\right)
\end{array}\right] d x^{*} d y^{*},
$$

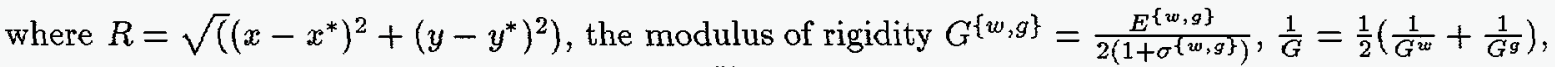
$\frac{\sigma}{G}=\frac{1}{2}\left(\frac{\sigma^{w}}{G^{w}}+\frac{\sigma^{g}}{G^{g}}\right)$, and $\kappa=\frac{G}{4}\left(\frac{\left(1-2 \sigma^{g}\right)}{G^{g}}-\frac{\left(1-2 \sigma^{w}\right)}{G^{w}}\right)$, with $E$ as the Young's Modulus of Elasticity, $\sigma$ as the Poisson's ratio and superscript $w$ and $g$ standing for the properties of the wheel and the ground, respectively.

Due to the elastic material flow with respect to a reference frame moving with the wheel, the net relative displacement of one body with respect to the other at a point in the area of contact is the sum of the gross rigid motion component and the relative elastic motion. The net relative velocity of one body with respect to the other at a point $(x, y) \in C_{e j}$ denoted $\dot{\mathbf{w}}\left(x, y, \nu_{x e j}, \nu_{y e j}, \nu_{\theta e j}\right)=\left(\dot{w}_{x e j}, \dot{w}_{y e j}\right)$ is

$$
\dot{w}_{x e j}=V_{e j}\left(\nu_{x e j}-y \nu_{\theta e j}\right)+\dot{u}_{x e j}(x, y, t)=V_{e j}\left(\nu_{x e j}-y \nu_{\theta e j}-\frac{\partial u_{x e j}}{\partial x}\right),
$$




$$
\dot{w}_{y e j}=V_{e j}\left(\nu_{y e j}+x \nu_{\theta e j}\right)+\dot{u}_{y e j}(x, y, t)=V_{e j}\left(\nu_{y e j}+x \nu_{\theta e j}-\frac{\partial u_{y e j}}{\partial x}\right)
$$

where steady-state assumption on the flow of material is assumed, i.e. $\frac{\partial \mathbf{u}_{e j}}{\partial t}=0$. Then, according to the law of Coulomb-Amontons

$$
\begin{array}{ll}
\text { - } & \left|\left(X_{e j}, Y_{e j}\right)\right| \leq \mu_{e j} Z_{e j}, \quad \text { and } \\
\text { - } \quad \dot{\mathbf{w}}_{e j} \neq 0 \Rightarrow\left(X_{e j}, Y_{e j}\right)=-\mu_{e j} Z_{e j} \frac{\dot{\mathbf{w}}_{e j}}{\left|\dot{\mathbf{w}}_{e j}\right|}
\end{array}
$$

where $\mu_{e j}$ is the co-efficient of friction at the wheel-ground interface of $e j^{t h}$ wheel.

The creep-force law problem is defined as:

Determine $\left(\lambda_{x e j}, \lambda_{y e j}, \lambda_{\theta e j}\right)$, the traction forces at the wheel-ground interface defined as

$$
\left(\lambda_{x e j}, \lambda_{y e j}, \lambda_{\theta e j}\right)=\iint_{C_{e j}}\left(X_{e j}, Y_{e j}, x Y_{e j}-y X_{e j}\right) d x d y
$$

so that equations (9), (10), and (11) are satisfied when the creepages $\left(\nu_{x e j}, \nu_{y e j}, \nu_{\theta e j}\right)$, the net load $N_{e j}=\iint_{C_{e j}} Z_{e j}(x, y) d x d y$ and the translational velocity $V_{e j}$ are known.

\subsection{Symmetry in Creep-Force Relation}

The problem of creep-force law as posed in (12) admits a symmetry relation that enables us to infer the traction forces on a special subset defined by no-lateral and no-angular slip.

Proposition 2 The traction symmetry relations

$$
\begin{aligned}
& \lambda_{x e j}\left(\nu_{x e j}, \nu_{y e j}, \nu_{\theta e j}\right)=\lambda_{x e j}\left(\nu_{x e j},-\nu_{y e j},-\nu_{\theta e j}\right), \\
& \lambda_{y e j}\left(\nu_{x e j}, \nu_{y e j}, \nu_{\theta e j}\right)=-\lambda_{y e j}\left(\nu_{x e j},-\nu_{y e j},-\nu_{\theta e j}\right), \\
& \lambda_{\theta e j}\left(\nu_{x e j}, \nu_{y e j}, \nu_{\theta e j}\right)=-\lambda_{\theta e j}\left(\nu_{x e j},-\nu_{y e j},-\nu_{\theta e j}\right),
\end{aligned}
$$

verify the creep-force law when the contact area $C_{e j}(x, y)=C_{e j}(x,-y)$.

Proof: The governing relations of creep-force law in equations (9), (10), and (11) can be shown to satisfy the following symmetry:

$$
\begin{aligned}
X_{e j}\left(x, y, \nu_{x e j}, \nu_{y e j}, \nu_{\theta e j}\right) & =X_{e j}\left(x,-y, \nu_{x e j},-\nu_{y e j},-\nu_{\theta e j}\right) \\
Y_{e j}\left(x, y, \nu_{x e j}, \nu_{y e j}, \nu_{\theta e j}\right) & =-Y_{e j}\left(x,-y, \nu_{x e j},-\nu_{y e j},-\nu_{\theta e j}\right) \\
Z_{e j}\left(x, y, \nu_{x e j}, \nu_{y e j}, \nu_{\theta e j}\right) & =Z_{e j}\left(x,-y, \nu_{x e j},-\nu_{y e j},-\nu_{\theta e j}\right) \\
u_{x}\left(x, y, \nu_{x e j}, \nu_{y e j}, \nu_{\theta e j}\right) & =u_{x}\left(x,-y, \nu_{x e j},-\nu_{y e j},-\nu_{\theta e j}\right) \\
u_{y}\left(x, y, \nu_{x e j}, \nu_{y e j}, \nu_{\theta e j}\right) & =-u_{y}\left(x,-y, \nu_{x e j},-\nu_{y e j},-\nu_{\theta e j}\right) \\
u_{z}\left(x, y, \nu_{x e j}, \nu_{y e j}, \nu_{\theta e j}\right) & =u_{z}\left(x,-y, \nu_{x e j},-\nu_{y e j},-\nu_{\theta e j}\right), \\
\frac{\partial u_{x}}{\partial x}\left(x, y, \nu_{x e j}, \nu_{y e j}, \nu_{\theta e j}\right) & =\frac{\partial u_{x}}{\partial x}\left(x,-y, \nu_{x e j},-\nu_{y e j},-\nu_{\theta e j}\right), \\
\frac{\partial u_{y}}{\partial x}\left(x, y, \nu_{x e j}, \nu_{y e j}, \nu_{\theta e j}\right) & =-\frac{\partial u_{y}}{\partial x}\left(x,-y, \nu_{x e j},-\nu_{y e j},-\nu_{\theta e j}\right) .
\end{aligned}
$$

The traction symmetry follows from the integral in equation (12).॰

Corollary 3 With no lateral and angular slip, the lateral and angular traction disappear when the contact area $C_{e j}(x, y)=C_{e j}(x,-y)$, i.e. $\lambda_{y e j}\left(\nu_{x e j}, 0,0\right)=\lambda_{\theta e j}\left(\nu_{x e j}, 0,0\right)=0$.

Unfortunately, the creep-force problem as posed obeys no other symmetry to exhibit any similar conclusion for the longitudinal traction $\lambda_{x}$. Kalker [Kalker 79], however, considered the following cases of quasi-identity: either the two elastic bodies are elastically similar, i.e. $E^{g}=E^{w}$, and $\sigma^{g}=\sigma^{w}$, or both are incompressible, i.e. $\sigma^{g}=\sigma^{w}=0.5$, then $\kappa$ in equation (9) is zero. Also, when one body, say a rubber wheel, is incompressible, and the other body is relatively rigid, i.e. $\sigma^{w}=0.5$, and 
$E^{w}<<E^{g}, \kappa$ is close to zero. When $\kappa$ is zero, the elastic strain and traction relations of equation (9) simplify in such a way that the problem of determining the vertical strain $u_{z}$, and therefore the vertical pressure distribution $Z(x, y)$, separates from that of the tangential problem of determining $u_{x}, u_{y}$ and the corresponding $X(x, y)$ and $Y(x, y)$. Let us call the elastic-traction relation derived from those of equations (9) with $\kappa=0$ the quasi-identical elastic-traction law and the corresponding creep-force law problem posed in (12) as the quasi-identical creep-force law. This separation of vertical and the tangential problem in quasi-identity allows several other symmetries in the creep-force law [Kalker 67] including a specialization of the Proposition 2 we proved earlier. We mention one other:

Proposition 4 (Kalker, 1967) The traction symmetry relations

$$
\begin{aligned}
& \lambda_{x e j}\left(\nu_{x e j}, \nu_{y e j}, \nu_{\theta e j}\right)=-\lambda_{x e j}\left(-\nu_{x e j}, \nu_{y e j}, \nu_{\theta e j}\right) \\
& \lambda_{y e j}\left(\nu_{x e j}, \nu_{y e j}, \nu_{\theta e j}\right)=\lambda_{y e j}\left(-\nu_{x e j}, \nu_{y e j}, \nu_{\theta e j}\right) \\
& \lambda_{\theta e j}\left(\nu_{x e j}, \nu_{y e j}, \nu_{\theta e j}\right)=\lambda_{\theta e j}\left(-\nu_{x e j}, \nu_{y e j}, \nu_{\theta e j}\right)
\end{aligned}
$$

verify the quasi-identity creep-force law when the contact area $C_{e j}(x, y)=C_{e j}(x,-y)$.

Corollary 5 (Kalker 1967) With no longitudinal slip, the longitudinal traction in quasi-identical problem disappears when the contact area $C_{e j}(x, y)=C_{e j}(x,-y)$, i.e. $\lambda_{x e j}\left(0, \nu_{y e j}, \nu_{\theta e j}\right)=0$.

\subsection{Kalker's Simplified Linear Theory}

The solution of the creep-force problem $\left(\lambda_{x e j}, \lambda_{y e j}, \lambda_{\theta e j}\right)$ as a function of $\left(\nu_{x e j}, \nu_{y e j}, \nu_{\theta e j}\right)$ with the elasticity-traction relations in equation (9) is called Exact Non-linear Theory. An analytical solution of the Exact Non-linear theory has not yet been possible. If, however, a linear elasticity-traction relation $\left(u_{x}, u_{y}\right)=\left(L_{x} X, L_{y} Y\right)$ is used with the quasi-identity assumption when $L_{x}$ and $L_{y}$ are fixed co-efficients, the resulting creep-force relation is given analytically. Consider first the normal problem. The solution is given by the Hertzian model [Kalker 79]. For twice continuously differentiable surfaces in contact, this area is elliptic in form. The pressure distribution $Z_{e j}\left(x^{\prime}, y^{\prime}\right)$ on the contact area $C_{e j}$ due to vertical load $N_{e j}$ on the wheel is given by $Z_{e j}\left(x^{\prime}, y^{\prime}\right)=\frac{3 N_{e j}}{2 \pi a b} \sqrt{1-\left(x^{\prime} / a\right)^{2}-\left(y^{\prime} / b\right)^{2}}$, where $x^{\prime}$ and $y^{\prime}$ are an appropriate choice of a co-ordinate system, $a$ and $b$ are the lengths of the major and the minor semi-axes of the elliptical contact area $C_{e j}$ determined as a function of the local radii of curvature of the two surfaces in contact, elastic properties of the two bodies and the total load $N_{e j}$. The tangential problem is based on the assumption that one of the principal semi-axis of the elliptical contact area is the direction of rolling - the $x$-axis of the slip co-ordinate frames. The problem of determining the strain $\left(u_{x}, u_{y}\right)$ and the corresponding tractions $(X, Y)$ assumes that the net slip $\dot{w}$ of equation (10) vanishes at all point in the contact area $C_{e j}$. The traction at the leading edge of contact area with respect to the direction of the rolling also vanishes. The analytical solution of the creep-force law thus obtained is:

$$
\left[\begin{array}{l}
\lambda_{x e j} \\
\lambda_{y e j} \\
\lambda_{\theta e j}
\end{array}\right]=\left[\begin{array}{ccc}
-a b G C_{11} & 0 & 0 \\
0 & -a b G C_{22} & -(a b)^{\frac{3}{2}} G C_{23} \\
0 & -(a b)^{\frac{3}{2}} G C_{32} & -(a b)^{2} G C_{33}
\end{array}\right]\left[\begin{array}{l}
\nu_{x e j} \\
\nu_{y e j} \\
\nu_{\theta e j}
\end{array}\right],
$$

where $C_{i j}$ 's are constants given in [Kalker 90] as a function of the combined Poisson's ratio $\sigma$ and the shape ratio $\frac{a}{b}$ of the semi-minor axes of the elliptical Hertz contact area of the two bodies. The resulting theory is called the Simplified Linear Theory due to Kalker [Kalker 67]. When the velocity $V_{e j}$ in equation (8) is zero, the steady state longitudinal and lateral traction vanishes and the spin traction moment can be calculated by elementary principles.

\section{Dynamic Model of Wheeled Mobile Robot}

The dynamic model of mobile robot is obtained by Euler-Lagrange formulation subject to the external forces applied at the actuated joints, and the forces at the wheel-ground interface. Let the vector of 
external (generalized) forces be $\tau=\left(0, \tau_{o c}, \tau_{\phi}, 0, \tau_{c}\right)$, where the three degrees of freedom of the base of the mobile robot $\mathbf{x}_{1}=(x, y, \theta)$ and the undriven direction of the omni-wheels are not directly actuated. The forces at the wheel-ground interface be denoted $\lambda_{x}, \lambda_{y} \lambda_{\theta}$ in the directions $x, y$, and $\theta$ of the slip co-ordinate frame of each of the wheels. Each of the $\lambda$ 's are $\left(N_{f}+N_{c}+N_{o c}+N_{o d}\right)$ long.

The generalized equations of motion is $\frac{d}{d t} \frac{\partial T}{\partial \mathbf{x}}-\frac{\partial T}{\partial \mathbf{X}}=\tau+\mathbf{J}_{x}^{T} \lambda_{x}+\mathbf{J}_{y}^{T} \lambda_{y}+\mathbf{J}_{\theta}^{T} \lambda_{\theta}$, where $T$ is the total energy of the system. Expanded into components, the equations of motion look like:

$$
\left[\begin{array}{ccccc}
I_{b} & 0 & 0 & 0 & 0 \\
0 & I_{o c} & 0 & 0 & 0 \\
0 & 0 & I_{\phi} & 0 & 0 \\
0 & 0 & 0 & I_{\bar{\phi}} & 0 \\
0 & 0 & 0 & 0 & I_{c}
\end{array}\right]\left[\begin{array}{c}
\ddot{\mathbf{x}}_{1} \\
\ddot{\mathbf{x}}_{2} \\
\ddot{\mathbf{x}}_{3} \\
\ddot{\mathbf{x}}_{4} \\
\ddot{\mathbf{x}}_{5}
\end{array}\right]=\left[\begin{array}{c}
0 \\
\tau_{o c} \\
\tau_{\phi} \\
0 \\
\tau_{c}
\end{array}\right]+\mathbf{J}_{x}^{T} \lambda_{x}+\mathbf{J}_{y}^{T} \lambda_{y}+\mathbf{J}_{\theta}^{T} \lambda_{\theta},
$$

where $I_{b}, I_{o c}, I_{\phi}, I_{\bar{\phi}}$ and $I_{c}$, are diagonal inertia matrices corresponding to the states $\mathbf{x}_{1}, \mathbf{x}_{2}, \mathbf{x}_{3}, \mathbf{x}_{4}$, and $\mathbf{x}_{5}$, respectively.

\section{Parameterization of Equations of Motion with Constraints}

\subsection{Lateral Constraints Only}

When lateral constraints are preserved, the kinematic constraints $\mathbf{J}_{y} \dot{\mathbf{x}}_{c}=0$ is imposed and the wheelground contact allows arbitrarily large forces to be transmitted along these constraints. The kinematic constraints are reduced to the following form:

$$
\begin{array}{r}
\mathbf{J}_{11}\left(\mathbf{x}_{5}\right) \hat{\mathbf{R}} \dot{\mathbf{x}}_{1}=0 \\
\mathbf{J}_{21}\left(\mathbf{x}_{2}\right) \hat{\mathbf{R}} \dot{\mathbf{x}}_{1}+\mathbf{J}_{22}() \dot{\mathbf{x}}_{2}=0 \\
\mathbf{J}_{33}\left(\mathbf{x}_{1}, \dot{\mathbf{x}}_{1}\right) \dot{\mathbf{x}}_{3}+\mathbf{J}_{34}\left(\mathbf{x}_{1}, \dot{\mathbf{x}}_{1}\right) \dot{\mathbf{x}}_{4}=0
\end{array}
$$

The first type of constraint arises from the fixed and centered wheels. The second type arises from those of the off-centered wheels and the third type from the omni-directional wheels. If $\mathbf{J}_{11}$ is of rank three, then any motion in the plane is impossible. The degree of mobility defined as the rank of the null space of the linear map $\mathbf{J}_{11}$ is the number of degrees of freedom the mobile robot has in the plane of motion. If this degree of mobility is three, then the mobile robot is called omni-directional since it has full mobility in the plane of motion. See Campion et. al. [CBD 93] for a classification based on the degree of mobility of the wheeled mobile robots. Consider a linear map $\Sigma=\Sigma\left(\mathbf{x}_{5}\right)$ whose columns span the null space of the mobility matrix $\mathbf{J}_{11}$, i.e., $\mathbf{J}_{11} \Sigma=0$. The velocity vector $\dot{\mathbf{x}}_{1}$ of the base of the mobile robot is restricted to lie in the following distribution:

$$
\dot{\mathbf{x}}_{1} \in \operatorname{span}\left\{\operatorname{col}\left(\hat{\mathbf{R}}^{T} \Sigma\right)\right\} \text {. }
$$

If the dimension of the null space of $\mathbf{J}_{11}$ is $m$, then consider an element $\mathbf{x}_{6}$ in $\mathcal{R}^{m}$ that parameterizes the mobility of the robot. In addition, assume that elements $\left(\mathbf{x}_{7}, \mathbf{x}_{8}\right)$ in $\mathcal{R}^{\left(N_{f}+N_{c}+N_{o c}+N_{o d}\right)} \times \mathcal{R}^{N_{c}}$ parameterize the velocities $\dot{\mathbf{x}}_{3}$ and $\dot{\mathbf{x}}_{5}$, respectively. The complete kinematic model of the wheeledmobile robot with no-slip in the lateral directions as in equations (15), (16), and (17) is parameterized by the vector $\mathbf{x}_{v}=\left(\mathbf{x}_{6}, \mathbf{x}_{7}, \mathbf{x}_{8}\right)$ given by the model

$$
\dot{\mathbf{x}}_{c}=\mathbf{P} \mathbf{x}_{v}
$$

where

$$
\mathbf{P}=\left[\begin{array}{ccc}
\hat{\mathbf{R}}^{T} \Sigma & 0 & 0 \\
-\mathbf{J}_{22}^{-1} \mathbf{J}_{21} \Sigma & 0 & 0 \\
0 & I & 0 \\
0 & -\mathbf{J}_{34}^{-1} \mathbf{J}_{33} & 0 \\
0 & 0 & I
\end{array}\right]
$$


The configuration parameters $\mathbf{x}_{c}=\left(\mathbf{x}_{1}, \mathbf{x}_{2}, \mathbf{x}_{3}, \mathbf{x}_{4}, \mathbf{x}_{5}\right)$ together with $\mathbf{x}_{v}=\left(\mathbf{x}_{6}, \mathbf{x}_{7}, \mathbf{x}_{8}\right)$, the independent parameterization of the velocities in equations (18), form the state space of the wheeled mobile robot. Let an element of this state space be denoted $\mathbf{x}=\left(\mathbf{x}_{c}, \mathbf{x}_{v}\right)$.

With a non-singular kinematic parameterization in equation (18), the equations of motion in equation (14) reduce to a set of first-order differential equations on the state space of the form $\dot{\mathbf{x}}=f(\mathbf{x})+g_{1}(\mathbf{x}) \tau_{o c}+g_{2}(\mathbf{x}) \tau_{\phi}+g_{3}(\mathbf{x}) \tau_{c}$. In components:

$$
\begin{gathered}
f(\mathbf{x})=\left[\begin{array}{c}
\hat{\mathbf{R}}^{T} \Sigma \mathbf{x}_{6} \\
-\mathbf{J}_{22}^{-1} \mathbf{J}_{21} \Sigma \mathbf{x}_{6} \\
\mathbf{x}_{7} \\
-\mathbf{J}_{34}^{-1} \mathbf{J}_{33} \mathbf{x}_{7} \\
\mathbf{x}_{8} \\
-\mathbf{I}_{1}^{-1} \mathbf{A} \mathbf{x}_{6} \\
\mathbf{I}_{2}^{-1}\left(\mathbf{I}_{21} \mathbf{I}_{1}^{-1} \mathbf{A}+\mathbf{B}\right) \mathbf{x}_{6} \\
0
\end{array}\right]+\left[\begin{array}{ccc}
0 & 0 & 0 \\
0 & 0 & 0 \\
0 & 0 & 0 \\
0 & 0 & 0 \\
0 & 0 & 0 \\
\mathbf{I}_{1}^{-1} & 0 & 0 \\
\mathbf{I}_{2}^{-1} \mathbf{I}_{21} \mathbf{I}_{1}^{-1} & \mathbf{I}_{2}^{-1} & 0 \\
0 & 0 & \mathbf{I}_{3}^{-1}
\end{array}\right] \mathbf{P}^{T}\left(\mathbf{J}_{x}^{T} \lambda_{x}+\mathbf{J}_{\theta}^{T} \lambda_{\theta}\right), \\
g_{1}(\mathbf{x})=\left[\begin{array}{c}
0 \\
0 \\
0 \\
0 \\
-\mathbf{I}_{1}^{-1} \Sigma^{T} \mathbf{J}_{21}^{T} \mathbf{J}_{22}^{-T} \\
-\mathbf{I}_{2}^{-1} \mathbf{I}_{21} \mathbf{I}_{1}^{-1} \Sigma^{T} \mathbf{J}_{21}^{T} \mathbf{J}_{22}^{-T} \\
0
\end{array}\right], \quad g_{2}(\mathbf{x})=\left[\begin{array}{c}
0 \\
0 \\
0 \\
0 \\
0 \\
0 \\
0 \\
\mathbf{I}_{2}^{-1} \\
0
\end{array}\right], \quad g_{3}(\mathbf{x})=\left[\begin{array}{c}
0 \\
0 \\
\mathbf{I}_{3}^{-1}
\end{array}\right],
\end{gathered}
$$

where $\operatorname{diag}\left(\mathbf{x}_{7}\right)$ is a diagonal matrix with the scalar elements of the state $\mathbf{x}_{7}$ on the diagonal, $\mathbf{Q}=\frac{d}{d t}\left(\hat{\mathbf{R}}^{T} \Sigma\right)=\mathbf{Q}\left(\mathbf{x}_{1}, \mathbf{x}_{5}, \mathbf{x}_{6}, \mathbf{x}_{8}\right),\left.\mathbf{Q}\right|_{\mathbf{x}=0}=0, \mathbf{T}=\frac{d}{d t}\left(-\mathbf{J}_{22}^{-1} \mathbf{J}_{21} \Sigma\right)=\mathbf{T}\left(\mathbf{x}_{2}, \mathbf{x}_{4}, \mathbf{x}_{5}, \mathbf{x}_{7}\right)$, and $\left.\mathbf{T}\right|_{\mathbf{x}=0}=0, \mathbf{K}=\frac{\partial}{\partial \dot{\mathbf{x}}_{1}}\left(\mathbf{J}_{34}^{-1} \mathbf{J}_{33}\right)=\mathbf{K}\left(\mathbf{x}_{1}, \mathbf{x}_{5}, \mathbf{x}_{6}\right), \mathbf{L}=\frac{\partial}{\partial \mathbf{x}_{1}}\left(\mathbf{J}_{34}^{-1} \mathbf{J}_{33}\right)=\mathbf{L}\left(\mathbf{x}_{1}, \mathbf{x}_{5}, \mathbf{x}_{6}\right), \mathbf{A}=\left(\Sigma^{T} \hat{\mathbf{R}} \mathbf{I}_{b} \mathbf{Q}-\right.$ $\left.\Sigma^{T} \mathbf{J}_{21}^{T} \mathbf{J}_{22}^{-1} \mathbf{I}_{o c} \mathbf{T}\right)=\mathbf{A}\left(\mathbf{x}_{1}, \mathbf{x}_{2}, \mathbf{x}_{4}, \mathbf{x}_{5}, \mathbf{x}_{7}\right), \mathbf{B}=\mathbf{J}_{33}^{T} \mathbf{J}_{34}^{-T} \mathbf{I}_{\bar{\phi}} \operatorname{diag}\left(\mathbf{x}_{7}\right)(\mathbf{K} \mathbf{Q}+\mathbf{L} \hat{\mathbf{R}} \Sigma)=\mathbf{B}\left(\mathbf{x}_{1}, \mathbf{x}_{4}, \mathbf{x}_{5}, \mathbf{x}_{7}\right)$, $\mathbf{I}_{1}=\Sigma^{T}\left(\hat{\mathbf{R}} \mathbf{I}_{b} \hat{\mathbf{R}}^{T}+\mathbf{J}_{21}^{T} \mathbf{J}_{22}^{-T} \mathbf{I}_{o c} \mathbf{J}_{22}^{-1} \mathbf{J}_{21}\right) \Sigma=\mathbf{I}_{1}\left(\mathbf{x}_{1}, \mathbf{x}_{2}, \mathbf{x}_{5}\right), \mathbf{I}_{21}=\mathbf{J}_{33}^{T} \mathbf{J}_{34}^{-T} \mathbf{I}_{\bar{\phi}} \operatorname{diag}\left(\mathbf{x}_{7}\right) \mathbf{K} \hat{\mathbf{R}}^{T} \Sigma=$ $\mathbf{I}_{21}\left(\mathbf{x}_{1}, \mathbf{x}_{5}, \mathbf{x}_{6}, \mathbf{x}_{7}\right)$, and $\mathbf{I}_{2}=\mathbf{I}_{\phi}+\mathbf{J}_{33}^{T} \mathbf{J}_{34}^{-T} \mathbf{I}_{\bar{\phi}} \mathbf{J}_{34}^{-1} \mathbf{J}_{33}=\mathbf{I}_{2}\left(\mathbf{x}_{1}, \mathbf{x}_{5}, \mathbf{x}_{6}\right), \mathbf{I}_{3}=\mathbf{I}_{c}=\mathbf{I}_{3}()$. In this form, we have assumed that $\lambda_{x}$ and $\lambda_{\theta}$, the components of the traction forces at the wheel-ground interface, are functions of the state, i.e., $\lambda_{x}=\lambda_{x}(\mathbf{x})$, and $\lambda_{\theta}=\lambda_{\theta}(\mathbf{x})$ (see Section 3).

\subsection{Lateral and Longitudinal Constraints}

The set of longitudinal constraints impose rolling with no-slip condition on the wheels of the mobile robot. We derive the conditions on the equations of motion if the longitudinal constraints, aside from the lateral ones, are also preserved. Recall that when longitudinal constraints are imposed in this manner, the equations of motion so obtained allow arbitrarily large wheel torque to be transmitted at the wheel-ground interface along the direction of their rolling.

The set of longitudinal constraints $\mathbf{J}_{x} \dot{\mathbf{x}}_{\boldsymbol{c}}=0$ is reduced to $\mathbf{J}_{x} \mathbf{P} \mathbf{x}_{v}=0$ on the space of motion of the mobile robot with the lateral constraints given by the map in equation (18). Consider the time-derivative of this constraint, i.e.

$$
\frac{\partial}{\partial \mathbf{x}}\left(\mathbf{J}_{x} \mathbf{P} \mathbf{x}_{v}\right) \dot{\mathbf{x}}=0
$$

To preserve this constraint on the state space, the set of first-order differential equations $\dot{\mathbf{x}}=f(\mathbf{x})+$ $g_{1}(\mathbf{x}) \tau_{o c}+g_{2}(\mathbf{x}) \tau_{\phi}+g_{3}(\mathbf{x}) \tau_{c}$ must satisfy them. With non-singular model, it can be shown that $\tau_{\phi}=$ $-\left(\mathbf{C} g_{2}\right)^{-1} \mathbf{C}\left(f(\mathbf{x})+g_{1}(\mathbf{x}) \tau_{o c}+g_{3}(\mathbf{x}) \tau_{c}\right)$, where the Jacobian of the constraints $\mathbf{C}(\mathbf{x})=\frac{\partial}{\partial \mathbf{x}}\left(\mathbf{J}_{x} \mathbf{P} \mathbf{x}_{v}\right)=$ $\mathbf{C}\left(\mathbf{x}_{1}, \mathbf{x}_{2}, \mathbf{x}_{5}, \mathbf{x}_{6}, \mathbf{x}_{7}\right)$. The first-order differential equations governing the motion of the mobile robot reduces to the form

$$
\dot{\mathbf{x}}=f^{\prime}(\mathbf{x})+g_{1}^{\prime}(\mathbf{x}) \tau_{o c}+g_{3}^{\prime}(\mathbf{x}) \tau_{c}
$$


where

$$
\begin{aligned}
& f^{\prime}(\mathbf{x})=f(\mathbf{x})+\left(0,0,0,0,0,0,-\mathbf{I}_{2}^{-1}\left(\mathbf{C} g_{2}\right)^{-1} \mathbf{C} f(\mathbf{x}), 0\right) \\
& g_{1}^{\prime}(\mathbf{x})=g_{1}(\mathbf{x})+\left(0,0,0,0,0,0,-\mathbf{I}_{2}^{-1}\left(\mathbf{C} g_{2}\right)^{-1} \mathbf{C} g_{1}(\mathbf{x}), 0\right), \\
& g_{3}^{\prime}(\mathbf{x})=g_{3}(\mathbf{x}) .
\end{aligned}
$$

Notice that since $\mathbf{J}_{x} \mathbf{P} \mathbf{x}_{v}$ is independent of the state $\mathbf{x}_{8}, \mathbf{C} g_{3}$ is zero, hence $g_{3}^{\prime}=g_{3}$.

\section{Can Lateral and Longitudinal Constraints be Preserved ?}

Consider the situation when neither lateral (sideways) nor the longitudinal (rolling) direction constraints are imposed on the equations of motion of a mobile robot with fixed, centered, off-centered, and omni-directional wheels, i.e. the traction forces in either of these directions are determined by the creep-force law of Section 3.

Theorem 6 In general, only mobile robots with off-centered wheels can preserve the lateral and longitudinal constraints.

Proof: The state-space is defined by the set of independent co-ordinates $\left(\mathbf{x}_{c}, \dot{\mathbf{x}}_{c}\right)=$ $\left(\mathbf{x}_{1}, \mathbf{x}_{2}, \mathbf{x}_{3}, \mathbf{x}_{4}, \mathbf{x}_{5}, \dot{\mathbf{x}}_{1}, \dot{\mathbf{x}}_{2}, \dot{\mathbf{x}}_{3}, \dot{\mathbf{x}}_{4}, \dot{\mathbf{x}}_{5}\right)$. The dynamic model of the mobile robot without lateral and longitudinal constraints imposed by the wheels is given by the equation (14). When the lateral and longitudinal constraints are preserved, it follows from the Corollaries 5 and 3 that wheel-ground traction forces $\lambda_{x}$ and $\lambda_{y}$ are zero.

Consider the off-centered wheels. If the lateral constraints are preserved, the longitudinal constraints in equation (5) for the off-centered wheels reduce to the form $\hat{\mathbf{J}}_{x 1 o c}\left(\mathbf{x}_{1}, \mathbf{x}_{2}\right) \dot{\mathbf{x}}_{1}+\hat{\mathbf{J}}_{x 3 o c}() \dot{\mathbf{x}}_{3}=0$. The Jacobian of the lateral and longitudinal constraints are:

$$
\begin{array}{r}
D_{\mathbf{x}_{1}}\left(\hat{\mathbf{J}}_{x 10 c} \dot{\mathbf{x}}_{1}\right) \dot{\mathbf{x}}_{1}+D_{\mathbf{x}_{2}}\left(\hat{\mathbf{J}}_{x 1 o c} \dot{\mathbf{x}}_{1}\right) \dot{\mathbf{x}}_{2}+\hat{\mathbf{J}}_{x 1 o c} \ddot{\mathbf{x}}_{1}+\hat{\mathbf{J}}_{x 3 o c} \ddot{\mathbf{x}}_{3}=0 \\
D_{\mathbf{x}_{1}}\left(\mathbf{J}_{21} \hat{\mathbf{R}} \dot{\mathbf{x}}_{1}\right) \dot{\mathbf{x}}_{1}+D_{\mathbf{x}_{2}}\left(\mathbf{J}_{21} \hat{\mathbf{R}} \dot{\mathbf{x}}_{1}\right) \dot{\mathbf{x}}_{2}+\mathbf{J}_{21} \hat{\mathbf{R}} \ddot{\mathbf{x}}_{1}+\hat{\mathbf{J}}_{22} \ddot{\mathbf{x}}_{2}=0
\end{array}
$$

The equations of motion (14) must preserve these constraints. It follows that the $\tau_{o c}$ and $\tau_{\phi o c}$ are determined uniquely as a function of the state. A similar construction for the fixed and centered wheels results in state dependent contraints that are not satisfied in general. For instance, only when $\left(\dot{\mathbf{x}}_{1}\right)_{3}$, the angular velocity of the base of the mobile robot, is zero, can the fixed and centered wheels preserve the lateral constraints. Another construction for the omni-directional wheels results in disparate $\tau_{\phi o d}$ arising from the lateral and the longitudinal constraints respectively. $\diamond$

Corollary 7 If a mobile robot with off-centered wheels preserves the lateral and the longitudinal constraints, then the state of rest is an equilibrium point.

Proof: At the state of rest, the spin is zero. Hence, by Corollary 3 , the angular traction vanishes. It is easy to verify that the drift term and the state-dependent inputs $\tau_{o c}$ and $\tau_{\phi o c}$ determined in the proof of Theorem 6 all vanish at the state of rest. $\diamond$

\section{Local Accessibility with Lateral Constraints}

Consider a control system of the form $\dot{\mathbf{x}}=f(\mathbf{x})+\Sigma_{i} g_{i}(\mathbf{x}) u_{i}$ defined on an open subset $V \subset \mathcal{S}$ of the state space where $f, g_{i}: V \rightarrow T V$ are smooth sections of the tangent bundle $T V$ with the control inputs $\left(u_{1}, \ldots, u_{p}\right) \in U \subset \mathcal{R}^{p}$. Let the flow of the dynamic system for a control input $\mathbf{u}:[0, t] \rightarrow U$ be denoted $\phi_{\mathbf{u}}: \mathcal{R} \times V \rightarrow V: \mathbf{x} \mapsto \phi_{\mathbf{u}}(t, \mathbf{x})$ and let $\mathcal{T}_{\phi_{\mathbf{u}}}(\mathbf{x})$ be the infimum of the times for which the flow at $\mathbf{x}$ is defined. An attainable set $\mathcal{A}(t, \mathbf{x})$ is a time-dependent reachable set defined as: $\mathcal{A}(t, \mathbf{x})=\left\{\mathbf{x}^{\prime} \in V \mid \exists \mathbf{u}:[0, t] \rightarrow U, \mathbf{x}^{\prime}=\phi \mathbf{u}(t, \mathbf{x})\right\}$. Let the forward projection $\mathbf{F P}(\mathbf{x})$ be the timeindependent reachable set defined as $\mathrm{FP}(\mathbf{x})=\left\{\mathbf{x}^{\prime} \in V \mid \exists \mathbf{u}:[0, t] \rightarrow U, \mathbf{x}^{\prime}=\phi_{\mathbf{u}}(t, \mathbf{x}), t \in \mathcal{T}_{\phi_{\mathbf{u}}(\mathbf{x})}\right\}$. Let $C^{\infty}(V, T V)$ be the space of smooth vector fields on $V$ with the Lie algebra defined by the standard Lie bracket $[$,$] of two vectorfields.$ 


\subsection{Accessibility/Controllability Algebra}

Let the accessibility algebra denoted $\mathcal{C}$ be the smallest subalgebra of the Lie algebra on $C^{\infty}(V, T V)$ containing the vector fields $f$ and the $g_{i}$ 's. If the $\operatorname{dim}(\mathcal{C}(\mathbf{x}))$ at a point $\mathbf{x}$ is equal to the dimension of the state space $V$, then the forward projection $\mathrm{FP}(\mathbf{x})$ contains an open neighborhood of $\mathbf{x}$. If the drift vector field $f$ of the control system vanishes, then this local accessibility result also implies local controllability [NV 90].

Theorem 8 Consider a mobile robot without off-centered wheels and preserving the rolling with no-slip condition restricted to the subset where the angular velocity of the plane of the centered wheels, if any, is zero. The dimension of the accessibility algebra $\mathcal{C}$ of the control system is zero at the state of rest in the subspace parameterizing the configuration and the velocity of the base of the mobile robot.

Proof: The accessibility algebra $\mathcal{C}$ is defined by brackets of the form

$$
\left[X_{k},\left[X_{k-1},\left[\ldots\left[X_{1}, X_{0}\right] \ldots\right]\right]\right],
$$

where $X_{i} \in\left\{f, g_{1}, g_{2}, \ldots\right\}$, for $i \in\{0,1,2, \ldots\}$. Our proof is by induction on the Lie brackets of increasing length of the aforementioned kind.

First, consider the equations of motion with lateral constraints in (19). The lagrangian forces due to the lateral constraints have been eliminated and therefore, arbitrarily large forces can be transmitted along this constraint. In addition, if we assume that the longitudinal constraints of rolling with no-slip conditions are preserved, then $\nu_{x e j}=0$ and we obtain a set of equations resulting from those in (20) by substituting $\lambda_{x e j}=0$ implied by the Corollary 5 .

There are no off-centered wheels. The state $\mathbf{x}_{4}$, the free-wheeling direction of the omni-directional wheels, is decoupled from the rest of the equations of motion. Hence, we drop the states $\mathbf{x}_{2}$ and $\mathbf{x}_{4}$, the control input $\tau_{o c}$ and by renumbering obtain the control equations whose functional dependency is as follows: $f^{\prime}(\mathbf{x})=f^{\prime 1}\left(\mathbf{x}_{1}, \mathbf{x}_{3}, \mathbf{x}_{4}\right), f^{\prime 2}(\mathbf{x})=f^{\prime 2}\left(\mathbf{x}_{5}\right), f^{\prime 3}(\mathbf{x})=f^{\prime 3}\left(\mathbf{x}_{6}\right), f^{\prime 4}(\mathbf{x})=f^{\prime 4}\left(\mathbf{x}_{1}, \mathbf{x}_{3}, \mathbf{x}_{4}, \mathbf{x}_{5}, \mathbf{x}_{6}\right)$, $f^{\prime^{5}}(\mathbf{x})=f^{\prime^{5}}\left(\mathbf{x}_{1}, \mathbf{x}_{3}, \mathbf{x}_{4}, \mathbf{x}_{5}, \mathbf{x}_{6}\right), f^{\prime 6}(\mathbf{x})=f^{\prime 6}\left(\mathbf{x}_{1}, \mathbf{x}_{3}, \mathbf{x}_{4}, \mathbf{x}_{5}, \mathbf{x}_{6}\right), g_{3}^{\prime j}(\mathbf{x})=0, j=1,2,3,4,5$, and $g_{3}^{6}(\mathbf{x})=$ $g_{3}^{\prime}()$. To recapitulate the states in the reduced notation, the state $\mathrm{x}_{1}$ parameterizes the configuration of the base of the mobile robot, $\mathbf{x}_{2}$ parameterizes the angular orientation of the wheels about their drive axes, $\mathbf{x}_{3}$ parameterizes the angular orientation of the plane containing the centered wheels, $\mathbf{x}_{4}$ parameterizes the set of independent velocities of the base of the mobile robot, $\mathbf{x}_{5}$ parameterizes the angular velocity of the wheels about their driven axes, and $\mathbf{x}_{6}$ parameterizes the angular velocity of the plane containing the centered wheels. Consider the following assumption of the induction:

1. Every bracket's first and fourth elements at the state of rest are zero.

Let $\left[f^{\prime}, g_{i}{ }^{\prime}\right]$ be a bracket of length one. The vector fields $f^{\prime}$ and $g_{3}^{\prime}$ are the zero level brackets of the accessibility algebra. The base level of induction is satisfied trivially. The induction proof on the brackets of length $k$ is divided into two parts: brackets with $f^{\prime}$ as the leading element and brackets with $g_{3}^{\prime}$ as the leading elements.

Consider an arbitrary bracket $\mathrm{Br}_{f^{\prime}}(k)$ of length $k$ with the leading term $f^{\prime}$ expressed in terms of $\operatorname{Br}(k-1)$ as

$$
\operatorname{Br}_{f^{\prime}}(k)=D \operatorname{Br}(k-1) f^{\prime}-D f^{\prime} \operatorname{Br}(k-1) .
$$

The drift $f^{\prime}\left|\mathbf{x = 0}=0, D_{j} f^{\prime 1}\right| \mathbf{x = 0}=0, j \neq 4$ and on the subset $\mathbf{x}_{6}=0$ corresponding to the zero angular velocity of the plane of the centered wheel $D_{j} f^{f^{4}} \mid \mathrm{x}=0=0, j \neq 4$. By the assumptions of the induction $\left.\mathrm{Br}^{1}(k-1)\right|_{\mathbf{x}=0}=0$ and $\mathrm{Br}^{4}(k-1) \mid \mathbf{x = 0}=0$, it follows that $\left.\mathrm{Br}^{1}(k)\right|_{\mathbf{x}=0}=0$ and $\left.\operatorname{Br}^{4}(k)\right|_{\mathbf{x}=0}=0$. Hence, the accessibility algebra does not contribute any element to the $\mathbf{x}_{1}$ - and $\mathbf{x}_{4}$-subspace.

Consider the case when the leading term of a bracket of length $k$ is $g_{3}^{\prime}$. An arbitrary bracket $\mathrm{Br}_{g_{3}^{\prime}}(k)$ of length $k$ with the leading term $g_{3}^{\prime}$ expressed in terms of $\operatorname{Br}(k-1)$ is

$$
\operatorname{Br}_{g_{3}^{\prime}}(k)=D \operatorname{Br}(k-1) g_{3}^{\prime}-D g_{3}^{\prime} \operatorname{Br}(k-1)
$$




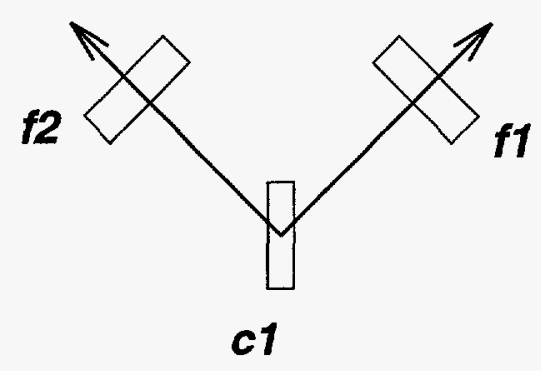

Figure 2: Controllability with centered wheel rotation.

Since $D g_{3}^{\prime} \equiv 0$, and the sixth term of $g_{3}^{\prime}$ is the only non-zero term, it follows that for $\operatorname{Br}_{g_{3}}(k)$ 's first and fourth term to be zero at the state of rest, the elements $D \operatorname{Br}(k-1)[1][6]$ and $D \operatorname{Br}(k-1)[4][6]$ must vanish at $\mathbf{x}=0$.

Lets consider the derivative $D_{i_{1}, \ldots, i_{l}} \operatorname{Br}_{X}(k), i_{q} \in\{1, \ldots, 6\}$.

$$
\begin{aligned}
& D_{i_{1}, \ldots, i_{l}} \operatorname{Br}_{X}(k)[i][j]=\Sigma_{\left.p \in 2^{\left\{i_{1}\right.}, \ldots, i_{l}\right\}}\left(\quad D_{p j} D \mathrm{Br}_{X}^{i}(k-1) D_{\bar{p}} X+D_{p} D \operatorname{Br}_{X}^{i}(k-1) D_{\bar{p} j} X\right. \\
& \left.-D_{p j} D X^{i} D_{\bar{p}} \operatorname{Br}(k-1)-D_{p} D X^{i} D_{\bar{p} j} \operatorname{Br}(k-1)\right) \text {, }
\end{aligned}
$$

where $2^{A}$ is a set of subsets of the set $A$ and for $p \in 2^{A}, \bar{p}$ is the complement of $p$ in $A$. The structure of $X, D_{p} X, D_{p} D X^{1}$, and $D_{p} D X^{4}$ at $\mathbf{x}=0$ for $X \in\left\{f^{\prime}, g_{3}^{\prime}\right\}$ determine those elements of the $D \mathrm{Br}(k-1)$ that are required to vanish for $D \mathrm{Br}_{X}(k)[i][j]$ to vanish. It can be verified that $\left.f^{\prime}\right|_{\mathbf{x}=0}=0,\left.D_{3} \ldots f^{\prime}\right|_{\mathbf{x}=0}=0,\left.D_{6} f^{\prime}\right|_{\mathbf{x}=0}$ has a non-zero element in the third row and is otherwise zero, $\left.D_{66} \ldots f^{\prime}\right|_{\mathbf{x}=0}=0$ with at least two derivatives with respect to $\mathbf{x}_{6},\left.D_{3 \ldots 6 \ldots} f^{\prime}\right|_{\mathbf{x}=0}=0,\left.D_{p} D f^{\prime j}\right|_{\mathbf{x}=0}$ for $j \in\{1,4\}, p \in 2^{\{3, \ldots, 6, \ldots\}}$ is zero if there is at least one derivative with respect to $\mathbf{x}_{6}$ and otherwise has a non-zero element in the fourth column and all derivatives of $g_{3}^{\prime}$ are zero.

For instance, for $D \mathrm{Br}_{f^{\prime}}(k)[1][6]$ to vanish, $D \mathrm{Br}(k-1)[1][3]$ and $D \mathrm{Br}(k-1)[4][6]$ must vanish. In general, our claim is that if the terms [1][3], [1][6], [4][3], and [4][6] of $D_{p} X, D_{p} D X$, for $X \in\left\{f^{\prime}, g_{3}^{\prime}\right\}$ at $\mathbf{x}=0$ and $p \in 2^{\{3, \ldots, 6, \ldots\}}$ of length depending on $k$, all vanish then the inductive assumptions are true. When this chain of dependency is followed until $k=0$, the brackets $\operatorname{Br}(0)$ must get instantiated to either the vector field $f^{\prime}$ or $g_{3}^{\prime}$ and we have already shown that such terms for $f^{\prime}$ or $g_{3}^{\prime}$ do indeed vanish at the state of rest. $\diamond$

Example 1 Consider a mobile robot with two fixed wheels and one centered orientable wheel as shown in Figure 2 with all constants such as the radius, lengths, and the inertias equal to one. The mobility of the mobile robot with this configuration of the wheels is one-rotation about the centered wheel. Let the lateral and the longitudinal constraints be preserved and consider the corresponding reduced form of the control equations given in the proof of the Theorem 8. The traction moment generated at the wheel ground interfaces of the centered and the fixed wheels given by the Kalker's Simplified model described in the Section 3.3 are $\lambda_{\theta f 1}=-f_{f 1} \frac{\mathbf{x}_{41}}{\left|\mathbf{x}_{41}\right|}, \lambda_{\theta f 2}=-f_{f 2} \frac{\mathbf{x}_{41} \mid}{\left|\mathbf{x}_{41}\right|}$, and $\lambda_{\theta c 1}=-f_{c 1} \frac{\mathbf{x}_{41}+\mathbf{x}_{61} \mid}{\left|\mathbf{x}_{41}+\mathbf{X}_{61}\right|}$, where $f_{f 1}, f_{f 2}$ and $f_{c 1}$ are positive constants. The control equations for this example is as follows: $f^{\prime}(\mathbf{x})=$ $\left(0,0, \mathbf{x}_{41}\right),{f^{\prime}}^{\prime 2}(\mathbf{x})=\mathbf{x}_{5}, f^{\prime 3}(\mathbf{x})=\mathbf{x}_{6},{f^{\prime}}^{4}(\mathbf{x})=-f_{f 1} \frac{\mathbf{x}_{41}}{\left|\mathbf{x}_{41}\right|}-f_{f 2} \frac{\mathbf{x}_{41}}{\left|\mathbf{x}_{41}\right|}-f_{c 1} \frac{\mathbf{x}_{41}+\mathbf{x}_{61} \mid}{\left|\mathbf{x}_{41}+\mathbf{x}_{61}\right|}, f^{\prime^{5}}(\mathbf{x})=(1,1,0) f^{\prime 4}$, $f^{\prime}(\mathbf{x})=-f_{c 1} \frac{\mathbf{x}_{41}+\mathbf{x}_{61} \mid}{\left|\mathbf{x}_{41}+\mathbf{x}_{61}\right|}+\tau_{c}$. It is easy to verify that for $f_{f 1}+f_{f 2} \neq f_{c 1}$, the set of differential equations has a solution such that the orientation of the mobile robot is changed from the state of rest when a torque $\tau_{c}>f_{c 1}$ is applied to turn the plane containing the centered wheel. This example shows that the restriction to zero angular velocity of the plane of the centered wheels in the Theorem 8 is necessary.

\section{Conclusion}

We have considered the dynamic model of motion of a mobile robot with an arbitrary combination of conventional fixed wheels, centered wheels, off-centered wheels, and omni-directional wheels. It is 
a standard practice in mechanics to reduce the number of independent variables describing the state of the mobile robot by considering that the wheels undergo rolling with no-slip motion. We, however, developed the dynamic model without incorporating the rolling with no-slip condition. Our model, therefore, included an analytical model of the traction forces generated by the rolling of wheels under a set of assumptions given in the Hypothesis 1 for the linearly elastic wheels.

We established that the lateral (sideways) constraints and the longitudinal (rolling direction) constraints imposed by the wheels can not, in general, be preserved by mobile robots with fixed, centered, and/or omni-directional wheels. The dynamic system of those mobile robots with off-centered wheels, however, is an autonomous system with no remaining control input such that the state of rest is an equilibrium point. Therefore, mobile robots with off-centered wheels must also slip.

Assuming that the lateral (sideways) slip of wheels for a straight line (large curvature) trajectory of the base of the mobile robot is likely to be small, we imposed the lateral constraints. We considered mobile robots with a combination of wheels of the conventional fixed, centered, or omni-directional type. We showed that the motion of the mobile robot has zero accessibility and controllability in the subspace of the position, orientation and the corresponding velocities of the base of the mobile robot as long as the angular orientation of the plane of the centered wheels has zero velocity. Therefore, the fixed, centered, and omni-directional wheels can not preserve the longitudinal (rolling direction) constraints. We also gave an example where the orientation of the base of the mobile robot can be changed by driving the angular orientation of the plane containing the centered wheels. This example showed the necessity of the minor condition of zero velocity of the plane of centered wheels on the zero controllability result. The base of a mobile robot with off-centered wheels can also change its configuration by a crab-like motion when the lateral constraints are imposed.

In summary, wheel slip is inevitable according to the proposed model. The wheel slip we establish in the paper may account for parts of the error in dead-reckoning - our ongoing work will address this issue.

\section{Acknowledgement}

This research was supported in part by the U.S. Air Force Material Command (AFMC) San Antonio Air Logistics Center, Robotics and Automation Center of Excellence (SA/ALC-RACE) under Interagency Agreement 2146-H055-A1 with the U.S. Department of Energy, in part by the Naval Air Warfare Center Aircraft Division under Interagency Agreement 2072-E123-A1 with the U.S. Department of Energy, under contract DE-AC05-96OR22464 with Lockheed Martin Energy Research Corp., and in part by an appointment to the Oak Ridge National Laboratory Postdoctoral Research Associates Program administered jointly by the Oak Ridge National Laboratory and the Oak Ridge Institute of Science and Education.

\section{References}

[AM 89] Alexander, J. C. and Maddocks, J. H. (1989) "On the Kinematics of Wheeled Mobile Robots," Int. J. Robotics Research, vol. 8(5), pp. 15-27.

[BG 95] Balakrishna, R. and Ghosal, A. (1995) "Modelling of Slip for Wheeled Mobile Robots," IEEE Tran. on Robotics and Automation, 11, 1, pp. 126-130.

[CBD 93] Campion, G., Bastin, G., and D'Andrea-Novel, B. (1993) "Structural Properties and Classification of Kinematic and Dynamic Models of Wheeled Mobile Robots," IEEE Int. Conf. on Robotics and Automation, Nice, pp. 462-469.

[Kalker 67] Kalker, J. J. (1967) "On the Rolling Contact of two Elastic Bodies in the presence of Dry Friction," Thesis, TU Delft, Faculty of Technical Mathematics and Informatics, The Netherlands.

[Kalker 79] Kalker, J. J. (1979) "Survey of Wheel-Rail Rolling Contact Theory," Vehicle System Dynamics, 5, pp. 317-358. 
[Kalker 90] Kalker, J. J. (1990) Three Dimensional Elastic Bodies in Rolling Contact, Kluwer Academic Publishers, Dordrecht, The Netherlands.

[Love 44] Love, A.E.H. (1944) A Treatise on the Mathematical Theory of Elasticity, 4th Ed., Dover Publications.

[NV 90] Nijmeijer H. and Van der schaft, A. J. (1990) Nonlinear Dynamical Control Systems, SpringerVerlag, New York.

[Sh 96] Shekhar, S. (1996) "Wheel Rolling Constraints and Slip in Mobile Robots," ORNL/TM, Oak Ridge National Laboratory, Oak Ridge, TN (in preparation).

\section{Appendix}

We give an instantiation of the kinematic constraints in the equation (4). Consider that the origin of the choice of co-ordinate system $\mathcal{O}_{e j}$ is given by $(l, \alpha)$ in polar co-ordinates in the choice of co-ordinate system $\mathcal{M}$. Similarly, the origin of the choice of co-ordinate system $\mathcal{M}_{e j}$ (or $\hat{\mathcal{M}}_{e j}$ ) is $\left(d, \beta-\frac{\pi}{2}\right.$ ) in the choice of co-ordinate system $\mathcal{O}_{e j}$ (see Figure 1). Let $\gamma$ be the angle that the direction of complimentary rolling $\bar{\phi}$ of an omni-directional wheel makes with the direction of $\phi$, the axis about which the wheel is driven. The three scalar components of the constraints in equation (4) are as follows:

$$
\begin{aligned}
& {[\cos (\delta) \quad \sin (\delta) \quad l \sin (\delta-\alpha)+d \cos (\alpha+\beta+\gamma-\delta)] \hat{\mathbf{R}} \dot{\mathbf{x}}_{1}-r \sin (\alpha+\beta-\delta) \dot{\phi}} \\
& \quad+d \dot{\beta} \cos (\alpha+\beta-\delta)-r^{\prime} \sin (\alpha+\beta+\gamma-\delta) \dot{\phi}, \\
& r \cos (\alpha+\beta-\delta) \dot{\phi}+r^{\prime} \dot{\bar{\phi}} \cos (\alpha+\beta+\gamma-\delta), \\
& {\left[\begin{array}{lll}
0 & 0 & 1
\end{array}\right] \hat{\mathbf{R}} \dot{\mathbf{x}}_{1}+\dot{\beta},}
\end{aligned}
$$

where $r$ is the radius of the wheel about the driven direction $\phi$, and $r^{\prime}$ is the radius of the omni-wheel about the complimentary direction $\bar{\phi}$ and $\delta$ is a quantity determined as explained in Section 2.3 from the choice of the transformations $\mathbf{h}_{e j}$ (by equating $[-\sin (\delta) \quad \cos (\delta) \quad l \cos (\delta-\alpha)+d \sin (\alpha+\beta+\gamma-\delta)] \hat{\mathbf{R}} \dot{\mathbf{x}}_{1}+d \dot{\beta} \sin (\alpha+\beta-\delta)$ to zero in this instantiation). For fixed wheels $d=0$ and $\beta$ is a constant, for centered wheels $d=0$. For centered and off-centered wheels $\beta$ is a state variable, a component of $\mathbf{x}_{5}$ and $\mathbf{x}_{2}$ respectively. For fixed, centered, and off-centered wheels, the component containing $\bar{\phi}$ does not appear and $\gamma=0$.

\section{DISCLAIMER}

This report was prepared as an account of work sponsored by an agency of the United States Government. Neither the United States Government nor any agency thereof, nor any of their employees, makes any warranty, express or implied, or assumes any legal liability or responsibility for the accuracy, completeness, or usefulness of any information, apparatus, product, or process disclosed, or represents that its use would not infringe privately owned rights. Reference herein to any specific commercial product, process, or service by trade name, trademark, manufacturer, or otherwise does not necessarily constitute or imply its endorsement, recommendation, or favoring by the United States Government or any agency thereof. The views and opinions of authors expressed herein do not necessarily state or reflect those of the United States Government or any agency thereof. 